\title{
Adaptive Multi-PHY IEEE802.15.4 TSCH in Sub-GHz Industrial Wireless Networks
}

\author{
Dries Van Leemput, Jan Bauwens, Robbe Elsas, Jeroen Hoebeke, Wout Joseph and Eli De Poorter \\ IDLab/WAVES - Department of Information Technology, Ghent University - imec \\ Technologiepark Zwijnaarde 126, 9000 Ghent, Belgium
}

\begin{abstract}
To provide wireless coverage in challenging industrial environments, IEEE802.15.4 Time-Slotted Channel Hopping (TSCH) presents a robust medium access protocol. Using multiple Physical Layers (PHYs) could improve TSCH even more in these heterogeneous environments. However, TSCH only defines one fixed-duration timeslot structure allowing one packet transmission. Using multiple PHYs with various data rates therefore does not yield any improvements because of this single-packet limitation combined with a fixed slot duration. We therefore defined two alternative timeslot structures allowing multiple packets transmissions to increase the throughput for higher data rate PHYs while meeting a fixed slot duration. In addition, we developed a flexible Link Quality Estimation (LQE) technique to dynamically switch between PHYs depending on the current environment. This paper covers a theoretical evaluation of the proposed slot structures in terms of throughput, energy consumption and memory constraints backed with an experimental validation, using a proof-of-concept implementation, which includes topology and PHY switching. Our results show that a $153 \%$ higher net throughput can be obtained with $84 \%$ of the original energy consumption and confirm our theoretical evaluation with a $99 \%$ accuracy. Additionally, we showed that in a real-life testbed of 33 nodes, spanning three floors and covering $2550 \mathrm{~m}^{2}$, a compact multi-PHY TSCH network can be formed. By distinguishing between reliable and high throughput PHYs, a maximum hop count of three was achieved with a maximum throughput of $219 \mathrm{kbps}$. Consequently, using multiple (dynamic) PHYs in a single TSCH network is possible while still being backwards compatible to the original fixed slot duration TSCH standard.
\end{abstract}

Index Terms-Multi-PHY, Time-Slotted Channel Hopping, Sub-GHz, IEEE802.15.4, Wireless Sensor Networks, Industrial Internet of Things

\section{INTRODUCTION}

The use of Wireless Sensor Networks (WSN) has proven to be valuable in a wide range of industrial applications, ranging from continuous process monitoring to time-critical emergency communication. It enables an efficient, cost effective and scalable alternative for wired solutions, with increased reliability and security due to multi-hop routing flexibility [4], [18]. However, industrial sites often consist of highly

Email addresses: dries.vanleemput@ugent.be (Dries Van Leemput), jan.bauwens2@ugent.be (Jan Bauwens), robbe.elsas@ugent.be (Robbe Elsas), jeroen.hoebeke@ugent.be (Jeroen Hoebeke),wout.joseph@ugent.be (Wout Joseph), eli.depoorter@ugent.be (Eli De Poorter) doi: https://doi.org/10.1016/j.adhoc.2020.102330

(c) 2020. This manuscript version is made available under the CC-BY-NC-ND 4.0 license http://creativecommons.org/licenses/by-nc-nd/4.0/

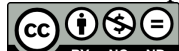

heterogeneous areas due to large metallic obstacles resulting in signal reflections, multi-path propagation and fading [26], [21], [24]. On top of that, targeted industrial applications have differing requirements in terms of latency, throughput, reliability and energy consumption. In order to allow sufficient flexibility to reuse the same technology in these heterogeneous areas for multiple applications, a robust and adaptive Medium Access Control (MAC) layer is needed.

The IEEE802.15.4 standard [1] is widely adopted for the challenging conditions that industrial environments pose on wireless networks, specifically whilst using the TSCH MAC mode to provide a solution against frequency selective fading. However, TSCH is usually defined in the Industrial, Scientific and Medical (ISM) $2.4 \mathrm{GHz}$ frequency band supporting a single PHY with a fixed Modulation and Coding Scheme (MCS) and data rate. When deployed in a highly heterogeneous environment, this can lead to places with sub-optimal throughput or isolated areas not being covered by the network. Furthermore, when multiple applications with differing requirements are needed inside the same network, it might prove difficult to find a suitable PHY.

This paper extends the conventional use of TSCH to be more adaptive and suitable for challenging industrial environments. Instead of using a fixed PHY and data rate, we use multiple sub-GHz PHYs because of the better propagation characteristics compared to $2.4 \mathrm{GHz}$ [25]. The use of multiple PHYs provides a trade-off between throughput and reliability and as a result, a representative PHY can be selected for each link depending on the environmental characteristics or use case. To remain compatible with the current specification and existing TSCH schedulers, a fixed timeslot is used irrespective of the used PHY, but multiple packets can be sent depending on the currently selected PHY. In order to also cope with dynamic link characteristics, we propose a LQE technique to assign a PHY to each link at run-time. Making use of the available PHYs for our development platform, the Zolertia RE-Mote featuring a CC1200 sub-GHz transceiver [22], we implemented a proof-of-concept adaptive multi-PHY TSCH implementation based on the Contiki-NG TSCH implementation. The implementation features a low data rate reliable $50 \mathrm{kbps}$ PHY and a high data rate $1 \mathrm{Mbps}$ PHY, and was used to test different timeslot structures in terms of throughput, energy consumption and topology.

In summary, the main contributions of this paper are as follows: 
- The design of a novel TSCH MAC protocol, backward compatible with the TSCH MAC specification, whereby a single slot can be used to send multiple packets depending on the currently selected PHY.

- Design of an adaptive link layer mechanism capable of automatically switching between PHYs depending on the current link quality.

- Providing mathematical formulas for calculating the net throughput and energy consumption for different PHY combinations assuming a predefined timeslot duration.

- Theoretical and experimental performance evaluation and validation of a proof-of-concept implementation featuring two PHYs.

The remainder of this paper is structured as follows: first related work on the use of multiple PHYs in IEEE802.15.4 and alternative TSCH schedulers is mentioned in Section $\Pi$ Next, some concepts concerning IEEE802.15.4-2015 TSCH, Orchestra and IPv6 Routing Protocol for LLNs (RPL) are highlighted in Section III Section IV covers the implementation of the adaptive TSCH enhancements with the defined timeslot structures, channel allocation and LQE process. The throughput, energy consumption, influence of Packet Reception Rate (PRR) and limited queues, switching mechanism and topology are evaluated in Section $\mathrm{V}$ Finally, Section $\mathrm{VI}$ shares some thoughts on future work and the paper is concluded in Section VII

\section{RELATED WORK}

\section{A. Multi-PHY (TSCH)}

Using different PHYs in IEEE802.15.4 (TSCH) has been explored previously. Table $\mathbb{1}$ lists other research works in comparison to our work in terms of theoretical and/or experimental evaluation, the presence of a dynamic MCS and whether or not they use a fixed or dynamic slot size (for research works applied to TSCH) and are thereby compatible with the current TSCH MAC specification and existing TSCH schedulers.

P. Tuset-Peiró et al. [24] cover extensive evaluation of the different Smart Utility Network (SUN) modulation schemes defined in the IEEE802.15.4-2015 standard (SUN-FrequencyShift Keying (FSK), SUN-Orthogonal Quadrature Phase-Shift Keying (OQPSK) and SUN-Orthogonal Frequency Division Multiplexing (OFDM)) including Received Signal Strength Indicator (RSSI), Clear Channel Assessment (CCA) and Packet Delivery Rate (PDR) measurements in an industrial environment. They highlight the need for additional schemes in order to meet industrial application requirements, and propose modulation diversity to meet these requirements. They see multiple PHYs as a similar means to create diversity compared to frequency diversity. In [12] the authors use these modulation schemes to propose three strategies to implement modulation diversity in low-power wireless networks in order to improve link reliability in terms of PDR and Required Number of Packet Transmissions (RNP). The authors used a real-world data set to perform simulations and show that, through the use of modulation diversity, the number of packet re-transmissions and thereby energy consumption and network congestion can drop significantly. Although these research works cover various modulation diversity strategies, a full experimental evaluation and an integration into TSCH lacks contrary to our work.

J. Munoz et al. [17] describe design considerations that need to be taken into account when using multiple PHYs in 6TiSCH networks [10]. Possible issues related to neighbor discovery and PHY capabilities, network formation, TSCH timeslot configuration, channel hopping sequence, resource allocation, packet size, and objective function are evaluated without proposing any solutions as the internet draft is purely informational. The mentioned difficulties range from the MAC layer up to the RPL routing layer. By contrast, we do provide some solutions and answers to the proposed design considerations, specifically for the MAC layer.

M. Brachmann et al. [7] proposes sub-GHz multi-PHY TSCH using the same development platform: CC1200 and Contiki-NG. Two implementations for multiple PHYs were evaluated: a single-template design using fixed slot sizes and a multi-template design with a dynamic slot size implementation. The former employs a timeslot tailored to the lowest data rate PHY and is experimentally evaluated to measure the differences between possible PHYs in terms of range and link symmetry. Higher data rate PHYs use the same timeslot without sending multiple packets and thereby miss out on possible throughput gains. This is in contrast to the multitemplate design, where lower data rate PHYs concatenate higher data rate PHY timeslots. This enables the researchers to emphasize the benefit of using multiple PHYs in the same network. However, the TSCH MAC specification does not define a dynamic slot size implying the multi-template design is not compatible. Our work builds on the single-template design while making use of unexploited time by sending multiple packets inside one timeslot. Also, a thorough theoretical evaluation is performed to calculate the net throughput and energy consumption for two extra timeslot structures. On top of that, we provide dynamic link adaptation to deal with heterogeneous environments.

Daneels et al. [8] describe a TSCH slot-bonding model in which slots of different durations and bandwidth, depending on the PHY layer, can be concatenated. Specifically, MCSs of the SUN-OFDM PHY layer offered in IEEE802.15.4g are used. Using a Mixed Integer Linear Program (MILP) model, the optimal MCS configuration is calculated which maximizes network-wide PDR while minimizing energy consumption. By making use of variable slot sizes, their design is not compatible with the current standard and the scheduling complexity is increased. As the research work mainly focuses on the extensive theoretical evaluation of the MILP scheduler, no proof-ofconcept implementation is provided. In contrast to our work, the capability to dynamically adapt PHYs assigned to links is not considered.

While modulation diversity is already being tackled in the above research works, we do provide several extensions to the topic. To the best of our knowledge, this is the first paper to 


\begin{tabular}{llllll} 
Work & Dynamic MCS & Theoretical eval. & Experimental eval. & Fixed/dynamic slots & TSCH MAC compatible \\
\hline [24], [12] Adaptive MCS & $\checkmark$ & $\checkmark$ & $\times$ & n.a. & n.a. \\
[17] Design considerations & $\times$ & $\checkmark$ & $\times$ & n.a. & n.a. \\
[7] Single-template & $\times$ & $\times$ & $\checkmark$ & fixed & $\checkmark$ \\
[7] Multi-template & $\times$ & $\times$ & $\checkmark$ & dynamic & $\times$ \\
[8] Slot bonding & $\times$ & $\checkmark$ & dynamic & $\times$ & \\
Our work & $\checkmark$ & $\checkmark$ & $\checkmark$ & fixed & $\checkmark$ \\
\hline
\end{tabular}

TABLE I: Comparison of existing multi-PHY (TSCH) research works with our work in terms of dynamic MCS, theoretical and/or experimental evaluation, fixed or dynamic slots, and TSCH MAC compatibility.

propose a multi-PHY TSCH design optimally making use of the provided timeslot duration by defining new multi-packet TSCH timeslots while remaining compatible with the current TSCH MAC specification and existing TSCH schedulers due to a fixed timeslot duration.

\section{B. TSCH Scheduling}

Next to extending TSCH with multiple MCSs, other works have also tried to enhance some commonly used TSCH scheduling functions in other ways. ALICE [16] is an Autonomous Link-based Cell Scheduling for TSCH. In this work, the authors provide an alternative for Orchestra [11] which performs better in terms of latency, throughput, routing stability and radio Duty-Cycle (DC). To facilitate this, instead of scheduling node IDs, directional links are used, multiple channel offsets are utilized and the cell allocation is changed every slotframe.

Apart from enhancing the Orchestra scheduler, Hamza et al. [13] introduce an extension to the TSCH minimal scheduling function. The scheduler predicts the transmitted control data for each pair of nodes in the next slotframe and anticipates in that way to control overhead. A distributed algorithm based on a Poisson process is used to predict schedule requirements. Their Enhanced Minimal Scheduling Function is able to decrease overhead, end-to-end latency and packet queue length.

\section{IEEE802.15.4 TSCH, ORCHESTRA \& RPL}

IEEE802.15.4 defines both PHY and MAC layer. Originally, a Maximum Transmission Unit (MTU) of 127 bytes MAC Protocol Data Unit (MPDU) was issued, restricting overhead and packet error rates. Depending on the addressing mode, the maximum payload length is 102 to 118 bytes. For the SUN PHYs, defined in the IEEE802.15.4-2015 standard, this MTU was extended to 2047 bytes [3]. The results in this paper are however restricted to the 127 bytes MTU, but can be equally well extended to the 2047 bytes MTU without loss of generality. IEEE802.15.4 packets are acknowledged by Enhanced Acknowledgements (EACKs) and Information Elements (IEs) can be appended to each packet or EACK to encapsulate necessary information such as drift compensation.

In 2012, the standard was extended with the IEEE802.15.4e2012 enhancement document [2] and defined several MAC modes including TSCH, later enrolled in the IEEE802.15.42015 standard and referred to as IEEE802.15.4-2015 TSCH mode. TSCH combines frequency division and time division multiplexing to form a reliable, predictable and low DC

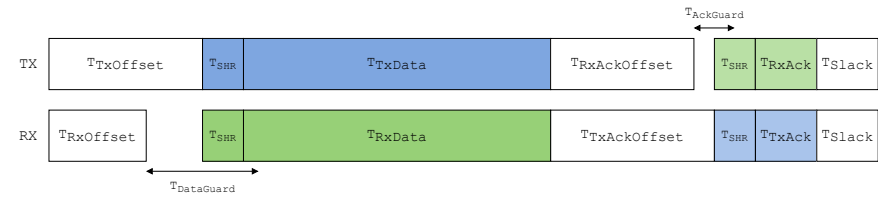

Fig. 1: Default TSCH timeslot timing. The timeslot allows for the transmission/reception of a single frame and the reception/transmission of an EACK. White timeslot sections indicate processing and arrows show synchronization guard bands between transmission and reception of a frame or EACK.

protocol and provides a solution for frequency selective fading. In TSCH, nodes send out Enhanced Beacons (EBs) to advertise the network, comprising slotframe, timeslot, synchronization and channel hopping information. After reception of an EB, nodes synchronize to the network and follow a periodically repeated slotframe consisting of a fixed number of timeslots. Nodes communicate using a link, defined as the pairwise assignment of a directed communication between nodes in a given timeslot on a given channel offset [3]. This offers an extra degree of freedom as in each timeslot different channel offsets can be used and provides a solution for frequency selective fading. Fig 1 illustrates the standard timing within a TSCH timeslot. Before the transmission/reception of a packet or EACK, processing has to be performed at both transmitter and receiver, indicated by TxOffset and RxOffset or TxAckOffset and RxAckOffset for an EACK. A DataGuard/AckGuard is installed in which the receiver listens for incoming packets to account for clock drift. Each timeslot is concluded with a Slack time. To calculate the frequency formula (1) is used, where ASN indicates the Absolute Slot Number, a shared variable by all nodes indicating the number of slots since the network creation. A channelOffset is assigned to each cell and $F$ represents a look-up table with size $n F r e q$, the amount of available frequencies.

$$
f=F\{(A S N+\text { channelOffset }) \% n F r e q\}
$$

As TSCH does not specify how to schedule links to nodes, an external scheduler must be used. We use Orchestra, a scheduler focused on dynamic, low-power IPv6 and RPL networks where nodes autonomously compute their own, local schedules based on their RPL parents and children [11]. 


\begin{tabular}{lllll}
\hline Throughput & MCS & Deviation & MI & Standard \\
\hline $1.2 \mathrm{kbps}$ & 2-GFSK & $3.99 \mathrm{kHz}$ & 1 & \\
$19.2 \mathrm{kbps}$ & 2-GFSK & $9.59 \mathrm{kHz}$ & 1 & \\
$50 \mathrm{kbps}$ & 2-GFSK & $24.95 \mathrm{kHz}$ & 1 & SUN-FSK \\
$100 \mathrm{kbps}$ & 2-GFSK & $49.90 \mathrm{kHz}$ & 1 & SUN-FSK/GFSK \\
$200 \mathrm{kbps}$ & 2-GFSK & $98.88 \mathrm{kHz}$ & 1 & \\
$500 \mathrm{kbps}$ & 2-GMSK & $124.82 \mathrm{kHz}$ & 0.5 & \\
$1 \mathrm{Mbps}$ & 4-GFSK & $399.17 \mathrm{kHz}$ & 1 & \\
\hline
\end{tabular}

TABLE II: CC1200 sub-GHz PHY implementations with throughput, MCS, frequency deviation and PHY standard.

Orchestra defines example schedules, one of which is used in our implementation: Sender-Based orchestra Schedule (SBS). In SBS, three slotframes are defined for different purposes with a variable amount of TSCH slots assigned to them: the EB slotframe is used to send EBs and a RPL broadcast slotframe can be used by all nodes in the network contending for channel access. The final unicast slotframe defines unicast slots between nodes. For each node, one shared TX link and a RX link for each parent and child is issued. In case slots of different slotframes collide, the slot associated with the slotframe of lowest priority is skipped in favour of the other slot.

In our proof-of-concept implementation, RPL is used as routing protocol. It is defined as a distance-vector routing protocol for low-power IPv6 networks [23]. It uses a Destination Oriented Directed Acyclic Graph (DODAG) structure giving a rank to each node using a predefined cost function. Since Orchestra is used as TSCH scheduler, RPL storing mode must be used implying that each node keeps a routing table to its children.

\section{ADAPTIVE MULTI-PHY TSCH}

This section (i) gives an overview of possible sub-GHz PHYs for the CC1200, (ii) discusses channel allocation, (iii) defines two extra slot structures next to the default TSCH timeslot, and (iv) describes the dynamic link adaptation mechanism.

\section{A. PHY's}

Table II lists seven possible sub-GHz PHY implementations for the CC1200 with corresponding data rate, MCS, frequency deviation, Modulation Index (MI), and which PHY specification it meets. Data rates range from $1.2 \mathrm{kbps}$ to $1 \mathrm{Mbps}$ and all PHYs use the 2-Gaussian Frequency-Shift Keying (GFSK) MCS except for $500 \mathrm{kbps}$ and $1 \mathrm{Mbps}$. $500 \mathrm{kbps}$ is also the only PHY with a MI deviating from 1 . The $100 \mathrm{kbps}$ PHY meets both SUN-FSK and GFKS requirements and $50 \mathrm{kbps}$ only the SUN-FSK requirements. It must be noted that these PHYs serve as an example for the CC1200, but that other standard compliant PHYs can be equally well incorporated.

\section{B. Channel allocation}

Unlike the $2.4 \mathrm{GHz}$ ISM band, where by default 16 equally sized TSCH channels can be defined, the allocation of channels in the sub-GHz spectrum is not a trivial task. Furthermore, these channels have a variable bandwidth as multiple PHYs are used within the same network. As pointed out in [19], the use of the sub-GHz spectrum is tightly regulated and sub- $\mathrm{GHz}$ technologies must comply to the Short-Range Device (SRD) regulations. Moreover, these regulations differ depending on the region of deployment which makes the implementation of a generic channel scheduler impossible. In the European Union (EU), both $863-870 \mathrm{MHz}$ and $873-920 \mathrm{MHz}$ spectra are divided in several bands with varying bandwidth $(50 \mathrm{kHz}$ up to $5 \mathrm{MHz}$ ), maximum TX power, DC restrictions and allowed applications. Therefore, a sub-GHz channel scheduler suited for multi-PHY TSCH in the EU should not only take the bandwidth of each PHY channel into account, but also the aforementioned variables. As will be discussed in Section V our proof-of-concept implementation makes use of a $50 \mathrm{kbps}$ and $1 \mathrm{Mbps}$ PHY. Texas Instruments recommends a channel width of $200 \mathrm{kHz}$ and $1667 \mathrm{kHz}$ respectively for these PHYs. Based on the results of [19], Table III] lists seven suitable sub$\mathrm{GHz}$ bands in which $200 \mathrm{kHz}$ and/or $1667 \mathrm{kHz}$ channels can be allocated. All bands are intended for non-specific SRDs or wideband data, have a maximum TX power of $14 \mathrm{dBm}$ or $27 \mathrm{dBm}$ and a DC limit of $2.7 \%$ provided a Polite Access (PA) mechanism is employed. As indicated in Table III 44 channels of $200 \mathrm{kHz}$ and four $1667 \mathrm{kHz}$ channels are possible. If no frequency overlap between channels of different PHYs is desired, this leaves either nineteen $200 \mathrm{kHz}$ channels and three $1667 \mathrm{kHz}$ channels, or nine $200 \mathrm{kHz}$ channels and four $1667 \mathrm{kHz}$ channels. This frequency overlap does not only result in inter-PHY interference, but also in a higher likelihood of DC saturation for a certain band. On the other hand, the allowance of frequency overlap has the consequence of higher frequency diversity for each PHY. This choice is therefore another variable in implementing a suitable channel scheduler.

The above example shows that channel allocation in the sub$\mathrm{GHz}$ spectrum for TSCH using multiple PHYs is possible, but not straightforward and moreover highly technology, implementation and region dependent. The definition of a generic worldwide applicable channel scheduler suitable for every scenario is therefore extremely complex, if not impossible.

\section{Timeslot structures}

To remain compatible with the TSCH MAC specification and existing TSCH schedulers such as Orchestra, our model always uses a fixed slot duration. This slot size can be chosen before network implementation and thus be customized to the network requirements. To cope with multiple PHYs, we extend the default TSCH timeslot with two slot structures able to send multiple packets. The following timeslot structures are supported within a single network:

- Default TSCH timeslot. This is similar to the default TSCH timeslot of Fig 1 as well as the sub-GHz alternative used in the single-template design of [7]. The timeslot allows for the transmission of one packet and reception of the associated EACK. At the beginning of each timeslot in TxOff set or RxOff set - some extra time is foreseen to re-configure the device to a new PHY. This results in 


\begin{tabular}{llllll} 
Nr. & Start freq & End freq & BW & $\mathbf{2 0 0} \mathbf{k H z}$ & $\mathbf{1 6 6 7} \mathbf{~} \mathbf{H z}$ \\
\hline 84 & $863 \mathrm{MHz}$ & $868 \mathrm{MHz}$ & $5000 \mathrm{kHz}$ & 25 & 3 \\
48 & $868 \mathrm{MHz}$ & $868.6 \mathrm{MHz}$ & $600 \mathrm{kHz}$ & 3 & 0 \\
50 & $868.7 \mathrm{MHz}$ & $869.2 \mathrm{MHz}$ & $500 \mathrm{kHz}$ & 2 & 0 \\
54 & $869.4 \mathrm{MHz}$ & $869.65 \mathrm{MHz}$ & $250 \mathrm{kHz}$ & 1 & 0 \\
$56 \mathrm{~b}$ & $869.7 \mathrm{MHz}$ & $870 \mathrm{MHz}$ & $300 \mathrm{kHz}$ & 1 & 0 \\
1 & $874 \mathrm{MHz}$ & $874.4 \mathrm{MHz}$ & $400 \mathrm{kHz}$ & 2 & 0 \\
5 & $917.4 \mathrm{MHz}$ & $919.4 \mathrm{MHz}$ & $2000 \mathrm{kHz}$ & 10 & 1 \\
\hline
\end{tabular}

TABLE III: Possible EU sub-GHz frequency bands for $200 \mathrm{kHz}$ and/or $1667 \mathrm{kHz}$ channels based on [19]
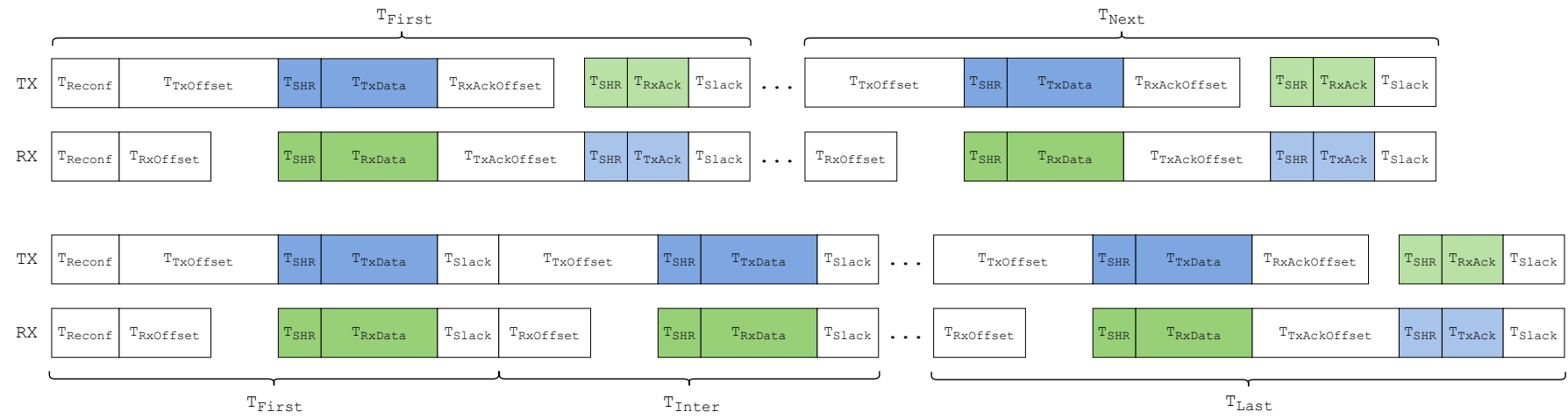

Fig. 2: Multi-packet TSCH timeslot structures. In the multi-ACK structure (top) each packet is being acknowledged in contrast to the single-ACK structure (bottom) where one EACK is sent at the end of the timeslot. The multi-ACK timeslot can be divided in the time to transmit/receive the first packet $T_{F i r s t}$ and a similar duration for subsequent packets $T_{\text {Next }}$. For the single-ACK structure, three durations are defined: $T_{\text {First }}, T_{\text {Inter }}$ and $T_{\text {Last }}$. Processing overhead is indicated by white timing sections.

$0.6 \mathrm{~ms}$ for the CC1200. By allowing to use the default TSCH timeslot structure, our model remains compatible with the TSCH MAC specification.

- Multi-ACK timeslot. In this slot structure, multiple packets can be sent if the selected PHY's data rate is high enough. Each packet transmission is acknowledged by the receiver and thereby a packet loss is instantly detected. In case of limited packet queues combined with a non ideal PRR, this can limit queue overflows as shown in Section $\mathrm{V}$ The slot structure is depicted in the top of Fig 2

- Single-ACK timeslot. The bottom of Fig 2 shows an alternative of the multi-ACK timeslot. Instead of acknowledging each packet, a single EACK is sent at the end of the timeslot in which every packet is acknowledged individually. This has the advantage of a higher net throughput since only one EACK has to be transmitted, as calculated in Section D

By defining two extra timeslot structures in which multiple packets can be sent, the available time inside a timeslot can be optimally used for each possible PHY in the network. In addition, a choice between a multi-ACK and single-ACK slot structure is provided. The optimal structure depends on the targeted scenario and will be discussed in Section $\mathrm{V}$

\section{Dynamic link adaptation}

In order to switch between PHYs at runtime, we define a dynamic link adaptation mechanism based on LQE. Each link is passively monitored at the receiving end to reduce packet overhead. To further restrict overhead, a RSSI hardware metric is chosen. Although RSSI has inherent drawbacks as a LQE metric in WSNs, such as temperature variability, and susceptibility to interference and multipath fading [6], [20], we use this estimator nonetheless because of its simplicity and readily availability on most chips. However, the proposed link adaptation mechanism and TSCH structures allow the use of different metrics without loss of generality. To associate the RSSI of a link to a possible PHY suited for the environment, Texas Instruments provides us with sensitivity limits for each CC1200 PHY [22]. If the RSSI value drops below the sensitivity limit of a certain PHY, communication is no longer possible using that PHY. As such, the PHY with the highest available data rate can be selected for each link. Using RSSI as a LQE metric permits to distinguish a link as either good or bad [5]. This implies that the sensitivity limits of the used PHYs in the network must be sufficiently separated to differentiate between PHYs. If PHYs with more convergent sensitivity limits are desired, more advanced LQE techniques must be used, at the cost of a higher computational complexity and therefore time and energy.

To avoid continuous switching between PHYs, a certain degree of stability must be established. We opted to use a hysteresis loop as a first measure, providing a configurable guard band in between the up and down switching RSSI thresholds. As a second measure, an Exponentially Weighted Moving Average (EWMA) filter is employed to filter incoming 
RSSI values according to (2).

$$
\hat{P}_{t}= \begin{cases}\mu_{t}, & t=1 \\ \hat{P}_{t-1} *(1-\alpha)-\alpha * \mu_{t}, & t>1\end{cases}
$$

$\mu_{t}$ represents the RSSI measurement, $\hat{P}_{t}$ the filter output and $\alpha[0,1]$ a smoothing parameter. By altering $\alpha$ we can opt to have a more stable estimator (smaller $\alpha$ ) or a more agile one (higher $\alpha$ ). This allows us to define an agile estimator when switching to a more reliable PHY and a stable estimator when switching to a less reliable PHY with higher data rate. This has the advantage of favouring the more reliable PHY to increase overall reliability.

The receiver makes a decision based on the acquired link quality information and feeds its decision back to the transmitter by encapsulating the PHY in the IE of the EACK. In the current TSCH implementation, the Time Correction IE encapsulates clock drift and NACK information. This is done by AND'ing a 16 bit two's-complement clock drift with Ox0FFF and OR'ing it with 0x8000 to encapsulate a possible NACK [3]. This results in 12 used bits for clock drift and 1 for NACK, leaving 3 bits unused. These bits can therefore be utilized to contain up to eight possible PHYs. In case the receiver decides to re-configure and the EACK gets lost, the transmitter and receiver will use a different PHY in subsequent communication. To prevent this, the transmitter will switch PHY after not receiving an EACK for four times, until both nodes use the same PHY. As such, nodes communicate using the same PHY that can dynamically change based on the current link quality.

\section{Evaluation}

The performance of each of the slot structures presented in Section $[\mathrm{IV}$ was thoroughly evaluated by calculating mathematical formulas representing both net throughput and energy consumption. The formulas apply to each PHY listed in Table III but equally well to every PHY that may be used in the presented slot structures. The acquired formulas were also experimentally validated with an addition of switching time and topology evaluation by means of a proof-of-concept implementation on the RE-Mote platform, featuring a $50 \mathrm{kbps}$ robust and a high data rate, but less robust, 1 Mbps PHY. Orchestra is used as TSCH scheduler, where the $50 \mathrm{kbps}$ PHY is used in the EB slotframe and RPL broadcast slotframe for enhanced robustness. The links associated with the unicast slotframe are adaptive and can switch between both PHYs depending on the link quality. The default TSCH timeslot of $50 \mathrm{kbps}$ will serve as slot size and both single-ACK as multiACK can be used in case of 1 Mbps.

Table IV] records several parameters that impact throughput and energy consumption. We can categorize the variables as either implementation specific variables (top half of Table IV) and PHY specific variables (bottom half of Table IV]. As can be seen, the timeslot size can be chosen before network deployment to meet heterogeneous network requirements, although this is restricted by the MTU and worst case PHY.
TxOffset and TxAckOffset are included in PHY specific variables as these tend to vary depending on the data rate for the chosen platform (CC1200) of this paper. These variations are likely to be explained by symbol-rate dependent processing [7]. When using a different platform, these variables could be classified as implementation specific variables. $T_{\text {Reconfig }}$ represents the re-configuration duration at the beginning of each timeslot. In our proof-of-concept implementation for the $\mathrm{CC} 1200$, this value was brought down to $600 \mu \mathrm{s}$.

\begin{tabular}{ll}
\hline Variable & Function \\
\hline$L_{\text {Payload }}$ & Payload length \\
$L_{\text {Data }}$ & $L_{\text {Payload }}+L_{P H R / M H R / M F R}$ \\
$L_{\text {Ack }}$ & ACK length \\
$T_{\text {DataGuard }}$ & Data RX guard time \\
$T_{\text {AckGuard }}$ & ACK RX guard time \\
$T_{\text {Reconf }}$ & Re-configuration time \\
$T_{\text {Slack }}$ & Slack time \\
$T_{T s}$ & Timeslot size \\
\hline$R_{\text {Data }}$ & Datarate \\
$L_{P H R / M H R / M F R}$ & PHY and MAC header and footer length \\
$L_{S H R}$ & Synchronization header \\
$T_{T x O f f s e t}$ & Offset before payload TX \\
$T_{T x A c k O f f s e t}$ & Offset before ACK TX \\
\hline
\end{tabular}

TABLE IV: Implementation specific (top half) and PHY specific (bottom half) variables impacting throughput and energy consumption. For platforms other than the CC1200, both $T_{T x A c k O f f s e t}$ and $T_{T x O f f s e t}$ can be classified as implementation specific variables.

\section{A. Single-link throughput}

Depending on the chosen slot structure, each PHY will have a different net throughput, smaller than the defined data rate $R_{\text {Data }}$ of that PHY. This is due to the processing overhead - indicated in white in Fig 1 and Fig 2 - EACK overhead, and PHY and MAC layer headers and footers. We define the single-link net throughput for each slot structure in (3).

$$
R_{\text {Net }}=\frac{8}{T_{T s}} \sum_{i=1}^{N} L_{\text {Payload }}^{i}
$$

$N$ represents the number of packets sent inside a timeslot of duration $T_{T s}$, with $L_{\text {Payload }}^{i}$ indicating the payload in bytes of the $i^{\text {th }}$ packet and the factor 8 accounting for the bytes to bits conversion. When we are interested in the maximum net throughput, we assume each packet has a maximum payload size of 118 bytes assuming the original 127 MTU of IEEE802.15.4 and (3) then reduces to (4), with $L_{\text {MaxPayload }}$ equalling 118. $L_{\text {MaxPayload }}$ can however also be adjusted to the 2047 bytes MTU without loss of generality.

$$
R_{\text {NetMax }}=\frac{8 N L_{\text {MaxPayload }}}{T_{T s}}
$$

Note that the actual (max) net throughput depends on the amount of slots per slotframe, being implementation specific. To calculate the actual net throughput, it suffices to replace $T_{T s}$ with the slotframe duration. However, even 
with a slotframe duration $>$ slot duration, the single-link net throughput could be achieved by making use of the burst link option, scheduling consecutive slots for a unicast link in case a burst of packets is expected. We therefore restrict ourselfs to making use of the single-link (max) net throughput.

For each slot structure, $N$ will have a different value, depending on the chosen slot size $T_{T s}$. It is imperative to say that $N_{D}=1$, for the default TSCH timeslot, provided $T_{T s}$ is chosen large enough, otherwise this falls back to zero. When considering the multi-ACK timeslot, $N_{M A c k}$ is given by (5), where a distinction is being made between the first packet transmission and the next transmissions as shown in (6). T Tirst follows the default TSCH timeslot time $T_{T s D}$ (including $T_{\text {Reconf }}$ ) associated with the currently selected PHY. For subsequent transmissions $\left(T_{\text {Next }}\right), T_{\text {Reconf }}$ can be left out since this is already performed at the beginning of the slot. Equation (5) calculates how many packets can be sent within $T_{T s}$ excluding $T_{\text {First }}$, hence the extra +1 . As this result could be negative, it is maximized to zero.

$$
\begin{gathered}
N_{M A c k}=\max \left(\left\lfloor\frac{T_{T s}-T_{\text {First }}}{T_{\text {Next }}}\right\rfloor+1,0\right) \\
T_{\text {First }}=T_{T s D} \\
T_{\text {Next }}=T_{T s D}-T_{\text {Reconf }}
\end{gathered}
$$

For the single-ACK timeslot, three types of packet transmissions can be distinguished, shown in (8). Since only one EACK is sent for the last packet transmission, $T_{T x A c k O f f s e t}$ and $T_{A c k}$ are not present in $T_{\text {First }}$ and $T_{\text {Inter. }}$. As was the case for the multi-ACK timeslot, $T_{\text {Reconf }}$ can be left out in all but the first transmissions. This leads us to (7).

$$
\begin{aligned}
& N_{S A c k}=\max \left(\left\lfloor\frac{T_{T s}-T_{\text {First }}-T_{\text {Last }}}{T_{\text {Inter }}}\right\rfloor+2,0\right) \\
& T_{\text {First }}=T_{\text {TsD }}-T_{\text {TxAckOffset }}-T_{\text {TxAck }} \\
& T_{\text {Inter }}=T_{T s D}-T_{\text {Reconf }}-T_{\text {TxAckOffset }}-T_{\text {TxAck }} \\
& T_{\text {Last }}=T_{T s D}-T_{\text {Reconf }}
\end{aligned}
$$

By using the above formulas, the (maximum) net throughput of each slot structure can be calculated given a predefined PHY and timeslot size.

Fig 3 shows the maximum net throughput as calculated in (4) for the $1 \mathrm{Mbps}$ PHY from Table II for each timeslot structure with increasing timeslot size up to $1 \mathrm{~s}$, which is the default timeslot length for the lowest PHY of $1.2 \mathrm{kbps}$ [7]. The default slot and multi-ACK slot coincide with the singletemplate and multi-template design of Brachmann et al. [7] respectively, although it must be noted that the multi-template design uses concatenated $1 \mathrm{Mbps}$ slots instead of a single multi-packet slot. As expected, the single-ACK slot structure provides the highest throughput for a given timeslot size, followed by the multi-ACK structure. Due to inherent processing overhead of the CC1200, the maximum net throughput stagnates to $253 \mathrm{kbps}$ and $165 \mathrm{kbps}$ for $1 \mathrm{~s}$ single-ACK and multiACK respectively, leading to a $153 \%$ increase when using

\begin{tabular}{llll}
\hline & 50 kbps default & 1 Mbps MACK & 1 Mbps SACK \\
\hline$T_{\text {theo }}$ & $31.32 \mathrm{kbps}$ & $156.60 \mathrm{kbps}$ & $219.24 \mathrm{kbps}$ \\
$T_{\text {exp }}$ & $31.17 \mathrm{kbps}$ & $156.27 \mathrm{kbps}$ & $217.31 \mathrm{kbps}$ \\
$P_{\text {val }}$ & $99.51 \%$ & $99.79 \%$ & $99.12 \%$ \\
\hline
\end{tabular}

TABLE V: Throughput experimental validation results. The single-link throughput can be validated for each timeslot structure with a validation percentage of $>99 \%$.

the single-ACK timeslot structure. This is in contrast to the theoretical upper bounds (only considering ACK overhead), which yield $887 \mathrm{kbps}$ and $797 \mathrm{kbps}$ for multi-ACK and singleACK respectively. As the majority of this processing overhead can be found in TxOffset and TxAckOffset $(2.2 \mathrm{~ms}$ and $1.9 \mathrm{~ms}$ for $1 \mathrm{Mbps}$ ), reducing these elements would increase the maximum net throughput. One way to do this is by loading the packet to the radio buffer in parallel to transmission, as implemented in the TAISC MAC protocol compiler and execution engine [14]. As a result, our two novel slot structures provide equal or even better throughput with better scheduler compatibility than the current state-of-the art sub-GHz TSCH timeslot structures.

The theoretical derived max net throughput was experimentally validated for each defined timeslot structure, using the possible slot structures implemented in the proof-of-concept implementation. The $50 \mathrm{kbps}$ PHY is used in the default slot structure, yielding a fixed timeslot size of $30.14 \mathrm{~ms}$. According to (5) and (7), this allows for a maximum of 5 and 7 packets for the multi-ACK and single-ACK structures respectively using the $1 \mathrm{Mbps}$ PHY. Using the maximum effective payload of 118 bytes and using (3), this lead to a maximum net throughput of $31.32 \mathrm{kbps}$ for the $50 \mathrm{kbps}$ default slot, $156.60 \mathrm{kbps}$ for the $1 \mathrm{Mbps}$ multi-ACK slot and $219.24 \mathrm{kbps}$ for a $1 \mathrm{Mbps}$ single-ACK slot. To validate these rates, two RE-Motes were connected with one sending a burst of packets for around 2.5 minutes and both missed packets and missed EACKs were taken into account. Table $\mathrm{V}$ lists the results of the average net throughput for ten measurements, with the corresponding validation percentage. As is clear from the table, the maximum net throughput for each slot structure can be experimentally validated with a $P_{v a l}>99 \%$.

\section{B. Energy consumption}

To detail the energy consumption of the presented slot structures in section IV, the duration of the various radio power modes were taken into account to provide us with formulas representative for every possible development platform and PHY. Since this will account for the majority of energy consumption, this simplification is justified. For a more detailed energy consumption modeling of dual-band devices comprising the CC1200 in TSCH and sub-GHz, the reader is referred to [9]. Equation (9] calculates the total charge for one timeslot, where $S$ indicates the set of radio states, $I^{s}$ the current consumption for a certain state and $T^{s}$ the time spent in that state. To calculate the total energy consumption in a 


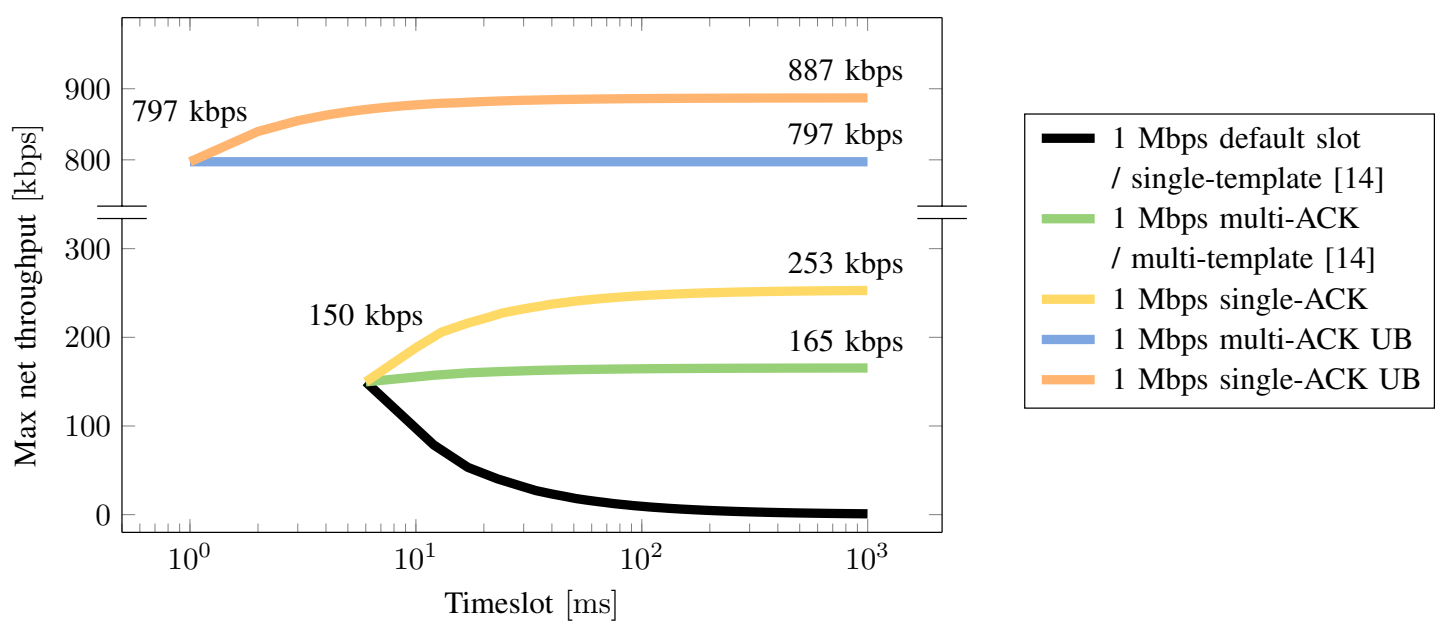

Fig. 3: Maximum net throughput obtained for each timeslot structure using both CC1200's 1 Mbps PHY and a no-processing 1 Mbps theoretical upper bound versus timeslot length up to $1 \mathrm{~s}$ (default slot length of lowest $1.2 \mathrm{kbps}$ PHY [7]). The throughput of the CC1200 PHY is clearly limited by the inherent processing overhead, resulting in a convergent throughput of only $165 \mathrm{kbps}$ and $253 \mathrm{kbps}$ for multi-ACK and single-ACK respectively. The single-ACK structure results in the highest throughput of $887 \mathrm{kbps}$ for a $1 \mathrm{~s}$ timeslot.

single timeslot, it suffices to multiply the total charge with the voltage on which the relevant platform is operating.

$$
Q_{T s}=\sum_{s \in S} T^{s} I^{s}
$$

Table VI lists the default TSCH timeslot sections of both a TX and a RX slot as depicted in Fig.1. Next to each timing section, the radio state is indicated. We distinguish four possible states: Idle, TX, RX or Listen. The value of $L_{S H R}$ represents the SHR field format and contains the preamble and Start Frame Delimiter (SFD). We assume a fixed $L_{S H R}$ value of 5 bytes according to the GFSK PHY specification, but this can be adjusted to meet certain other PHY requirements. Other variables to calculate the time of each section can be found in Table IV As pointed out above, $T_{T x O f f s e t}$ and $T_{T x A c k O f f s e t}$ are variable for our development platform, but might be fixed for any other platforms. This allows us to calculate the radio state times for a RX and TX slot in (10) - (15) which are applicable to all three defined slot structures and where for $T_{a}^{s}$ the suffix $a$ represents the performed action and $s$ the radio state the radio is currently in.

The most decisive state times are the transmission or reception of the packet, calculated in (10), where $T_{\text {Data }}^{T X}$ and $T_{\text {Data }}^{R X}$ represent these state times for a transmission and reception respectively. These are also the only terms that are not fixed, since they depend on the variable payload size of each packet. In case the maximum energy consumption is desired, it suffices to replace each $L_{\text {Data }}^{i}$ with $L_{\text {DataMax }}$ of 128 bytes, assuming a one byte PHY Header (PHR) conform the GFSK PHY specification. The variable $N$ again indicates the amount of packets sent within one timeslot and depends on the timeslot

\begin{tabular}{|c|c|c|}
\hline Action & Time & Radio state \\
\hline$T_{\text {Reconf }}$ & $600 \mu \mathrm{s}$ & Idle \\
\hline$T_{T x O f f s e t}$ & variable & Idle \\
\hline$T_{S H R}$ & $8^{*} L_{S H R} / R_{D a t a}$ & $\mathrm{TX}$ \\
\hline$T_{\text {Data }}$ & $8^{*} L_{\text {Data }} / R_{\text {Data }}$ & $\mathrm{TX}$ \\
\hline$T_{R x A c k O f f s e t}$ & $T_{T x \text { AckOffset }}-T_{\text {AckGuard }} / 2$ & Idle \\
\hline$T_{\text {AckListen }}$ & $T_{\text {AckGuard }} / 2$ & Listen \\
\hline$T_{S H R}$ & $8^{*} L_{S H R} / R_{\text {Data }}$ & $\mathrm{RX}$ \\
\hline$T_{A c k}$ & $8^{*} L_{A c k} / R_{D a t a}$ & $\mathrm{RX}$ \\
\hline$T_{\text {Slack }}$ & $T_{\text {Slack }}$ & Idle \\
\hline$T_{\text {Reconf }}$ & $600 \mu \mathrm{s}$ & Idle \\
\hline$T_{R x O f f s e t}$ & $T_{\text {TxOffset }}-T_{\text {DataGuard }} / 2$ & Idle \\
\hline$T_{\text {DataListen }}$ & $T_{\text {DataGuard }} / 2$ & Listen \\
\hline$T_{S H R}$ & $8^{*} L_{S H R} / R_{D a t a}$ & $\mathrm{RX}$ \\
\hline$T_{\text {Data }}$ & $8^{*} L_{\text {Data }} / R_{\text {Data }}$ & $\mathrm{RX}$ \\
\hline$T_{T x A c k O f f s e t}$ & variable & Idle \\
\hline$T_{S H R}$ & $8^{*} L_{S H R} / R_{\text {Data }}$ & $\mathrm{TX}$ \\
\hline$T_{A c k}$ & $8^{*} L_{A c k} / R_{D a t a}$ & $\mathrm{TX}$ \\
\hline$T_{\text {Slack }}$ & $T_{\text {Slack }}$ & Idle \\
\hline
\end{tabular}

TABLE VI: TX slot (top half) and RX slot (bottom half) timing and radio states.

structure.

$$
\begin{aligned}
T_{\text {Data }}^{T X} & =T_{\text {Data }}^{R X} \\
& =\frac{8}{R_{\text {Data }}} \sum_{i=1}^{N}\left(L_{S H R}+L_{\text {Data }}^{i}\right)
\end{aligned}
$$

Equation (11) calculates the EACK transmission and reception time. It almost equals (10), except that it is fixed due to the EACK length $L_{A c k}$ and that $N$ is replaced by $M$, the number of EACKs sent/received within one timeslot. The latter 


\begin{tabular}{lr}
\hline Radio state & Current [mA] \\
\hline CC1200 Idle & 1.5 \\
CC1200 TX $(14 \mathrm{dBm})$ & 46 \\
CC1200 Listen & 23.5 \\
CC1200 RX & 23.5 \\
\hline
\end{tabular}

TABLE VII: Radio states current draw.

is calculated in 12 .

$$
\begin{aligned}
T_{A c k}^{R X} & =T_{A c k}^{T X} \\
& =\frac{8 M}{R_{\text {Data }}}\left(L_{S H R}+L_{A c k}\right) \\
M & = \begin{cases}N_{D}, & \text { single-ACK } \\
N, & \text { otherwise }\end{cases}
\end{aligned}
$$

The time spent listening for a packet or EACK $T_{\text {Listen }}$ depends on both $T_{\text {AckGuard }}$ and $T_{\text {DataGuard }}$ for (13) and (14) respectively. Here we assumed the receiver does not have to wait for the packet or EACK and receives it instantly, thereby reducing the listen time to half the guard time for each case.

$$
\begin{aligned}
T_{\text {AckListen }}^{\text {Listen }} & =\frac{M}{2} T_{\text {AckGuard }} \\
T_{\text {DataListen }}^{\text {Listen }} & =\frac{N}{2} T_{\text {DataGuard }}
\end{aligned}
$$

The remaining time for each slot structure is spent in the idle state, so it suffices to substract the previous state durations from the total timeslot time $T_{T s}$, as shown in (15).

$$
T^{I d l e}=T_{T s}-T^{T X}-T^{R X}-T^{\text {Listen }}
$$

Fig 4 depicts the charge per bit for the $1 \mathrm{Mbps}$ PHY and both multi-packet TX and RX slots with increasing timeslot size. In this figure, all packets assume to have the maximum length of 128 bytes and the CC1200 radio states are listed in Table VII Here, the listen current draw is equal to the RX current draw, but this can be brought down to $0.5 \mathrm{~mA}$ if the $\mathrm{RX}$ sniff mode is used [22]. It is noticeable that, apart from resulting in a higher maximum net throughput, the single-ACK slot structure also leads to a lower energy consumption per bit: $84 \%$ for a TX and $86 \%$ for a RX slot. Similar to Fig 3 , the energy consumption per bit stagnates to a constant value.

As pointed out in Section IV] in a multi-ACK timeslot the transmitter can decide to postpone remaining packets to the next slot if the receiver is not receiving its packets to reduce energy consumption. In a non-ideal asymmetric link, this can result in a lower energy consumption per bit compared to the single-ACK timeslot. Fig.... shows the charge per bit for both multi-ACK and single-ACK timeslots for a $1 \mathrm{Mbps}$ PHY using a $30.14 \mathrm{~ms}$ timeslot.

\section{PRR and constrained memory}

Up until now we have assumed a perfect PRR with no constraints regarding available memory. However, most devices used in WSNs have some serious memory constraints [18]. With the extension of multi-packet timeslots, it may prove to

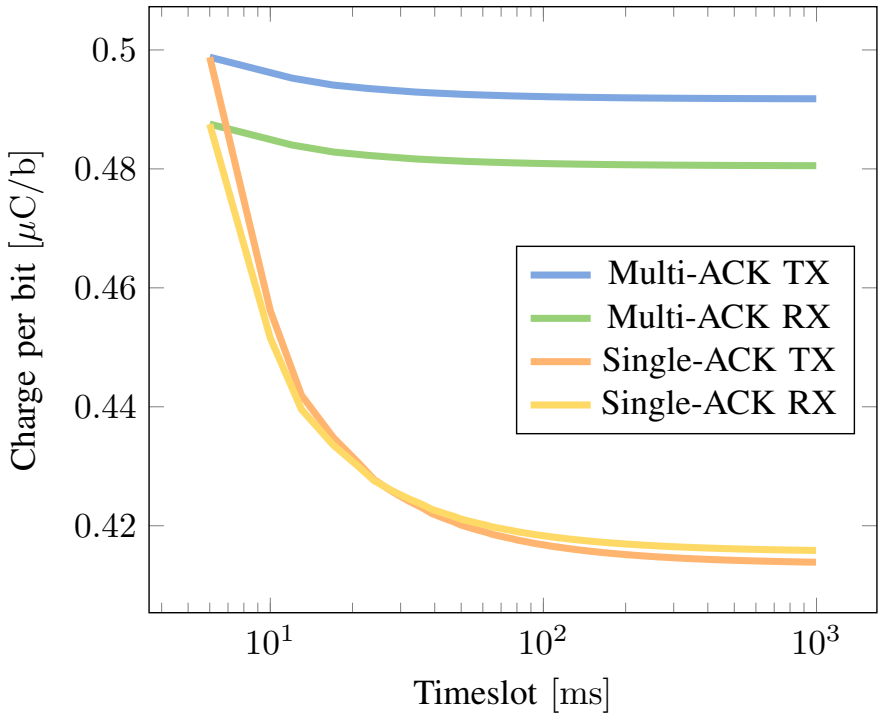

Fig. 4: Energy consumption per bit for the multi-ACK and single-ACK structures and $1 \mathrm{Mbps}$ PHY versus timeslot length up to $1 \mathrm{~s}$. The energy consumption for the single-ACK structure is clearly lower for both TX (84\%) and RX (86\%) slots. As was the case for the net throughput, the energy consumption per bit converges for both slot structures.

be a challenging task to allocate a sufficiently large packet queue for in and outgoing packets, since they are processed at the end of a timeslot to reduce timing overhead. This implies the size of a RX and TX queue has to be at least equal to the amount of packets that can be sent within that timeslot. Furthermore, each node has to keep both a RX and TX queue for the maximum allowed of neighbours in the network. Assuming a maximum packet length of 128 bytes, a RX/TX queue of eight packets and the maximum number of neighbours to be ten, this results in $20 \mathrm{kB}$ of Random Access Memory (RAM) allocated exclusively for RX and TX queues. As a reference, the Zolertia RE-Mote offers a RAM of $32 \mathrm{kB}$.

The limited queue size combined with a non-perfect PRR will inevitably result in queue overflows and as a result lost packets. In this light, although the single-ACK timeslot structure outperforms the multi-ACK structure on both throughput and energy consumption, a multi-ACK structure might be more resistant to queue overflows in certain scenarios. To illustrate this, Fig 5 shows the Packet Loss Rate (PLR) due to queue overflows for a multi-ACK timeslot structure able to send five packets and a single-ACK structure supporting seven packets per timeslot, in line with our proof-of-concept setup. In this simulation, we assumed a queue buffer of eight packets and a packet generation rate of two packets per timeslot. From Fig 5 it is clear that in between a PRR of $64 \%$ and $92 \%$, the multiACK structure outperforms the single-ACK structure. This can be explained by the fact that in the single-ACK structure, an EACK miss results in seven packets to be re-entered in the TX queue, while for the multi-ACK structure this only results in a single re-entry. It must however be noted that the 
above example is only a specific scenario, but it shows that in some cases multi-ACK outperforms single-ACK in terms of queue overflows. Furthermore, in case it is not possible to foresee sufficiently large queue sizes to cope with the amount of packets sent within a single-ACK timeslot, a multi-ACK timeslot structure provides a valuable alternative.

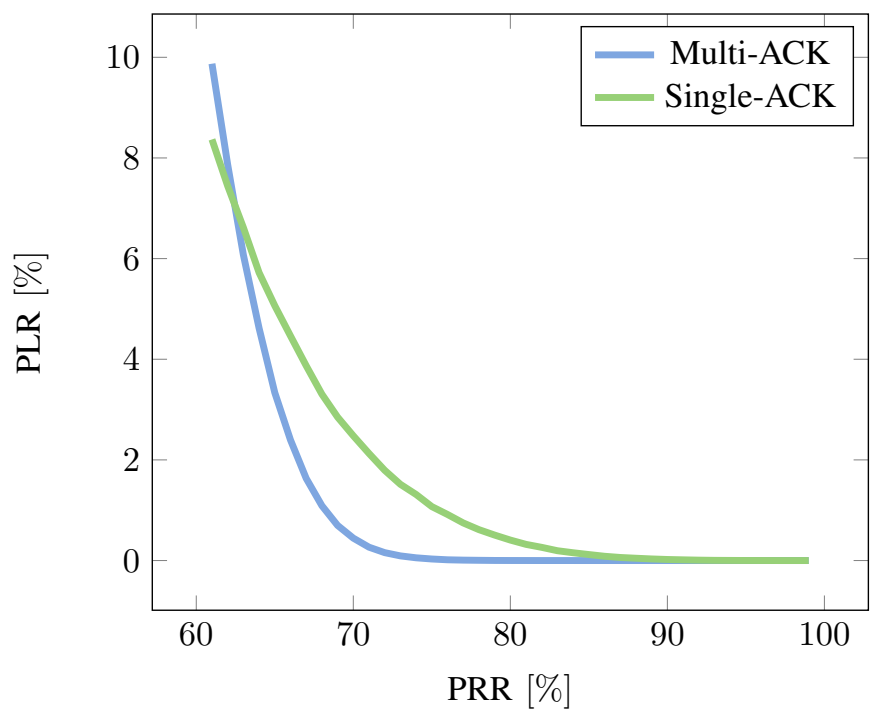

Fig. 5: PLR due to queue overflows vs PRR for a five packets per timeslot multi-ACK and seven packets per timeslot singleACK structure. The queue can hold eight packets and every timeslot two packets are generated. In between $64 \%$ and $92 \%$, the multi-ACK structure outperforms the single-ACK structure.

\section{Switching mechanism}

In order to reflect the current link quality of a link to the used PHY, the dynamic link adaptation technique discussed in section [V] was implemented in our proof-of-concept implementation, making use of the EWMA filter of (2). To switch from $50 \mathrm{kbps}$ to $1 \mathrm{Mbps} \alpha=0.5$, is chosen with a threshold of $-65 \mathrm{dBm}$ and $\alpha=0.75$, with a $-70 \mathrm{dBm}$ threshold in the opposite direction. This creates a $5 \mathrm{~dB}$ hysteresis guard band and an extra $12 \mathrm{~dB}$ band between the 1 Mbps sensitivity limit of $-82 \mathrm{dBm}$, which was found to be necessary in our experiments to ensure a reliable communication. Fig 6 shows both raw RSSI and the current EWMA filter output together with the instantaneous single-link throughput of a single burst unicast link for a RSSI increase resulting in a re-configuration to $1 \mathrm{Mbps}$ (6a) and RSSI decrease following a re-configuration to $50 \mathrm{kbps}$ (6b). The shift in RSSI was accomplished by altering the TX power. One can clearly see the difference in agility between the two scenarios due to a different choice of $\alpha$. In Fig 6a, although the first raw RSSI value after the increase is about $12 \mathrm{~dB}$ above the switching threshold, the EWMA filter output does not yet trigger a re-configuration to favour the more reliable PHY. Instead, it takes another slot to reach the switching threshold resulting in a total duration of three timeslots or $90.42 \mathrm{~ms}$ (in burst mode). In contrast,

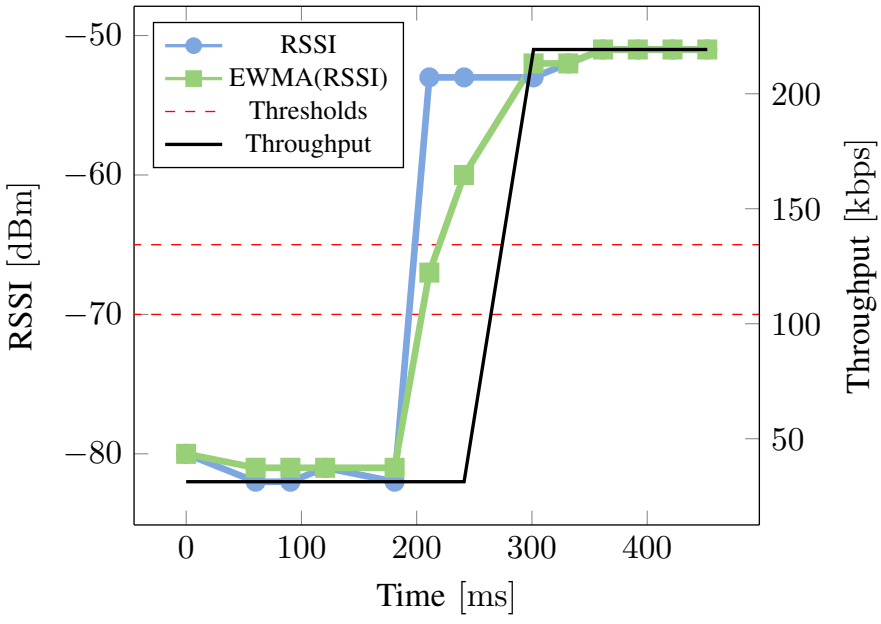

(a) Re-configuration from $50 \mathrm{kbps}$ to $1 \mathrm{Mbps}$

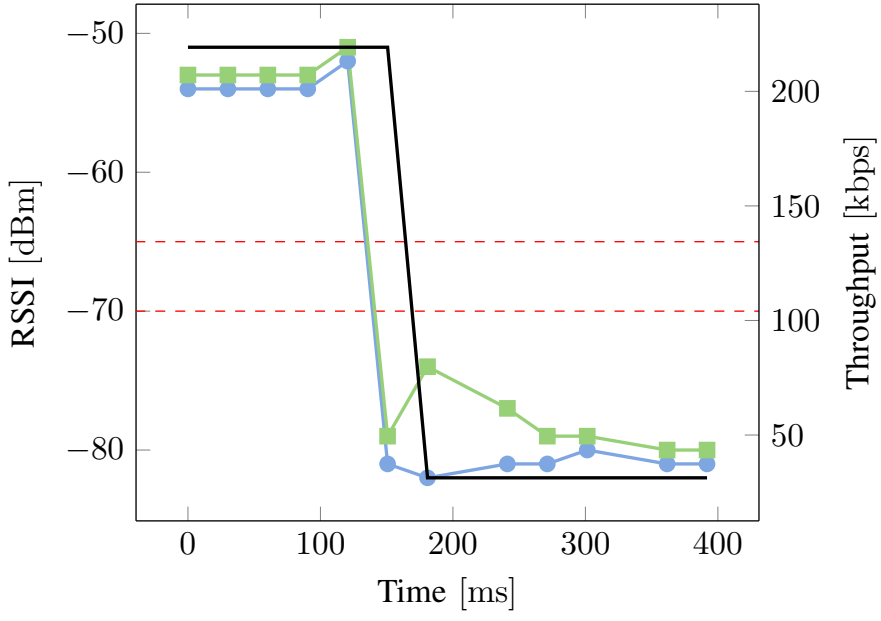

(b) Re-configuration from $1 \mathrm{Mbps}$ to $50 \mathrm{kbps}$

Fig. 6: Experimental switching mechanism evaluation of both a signal increase and decrease with accompanying RSSI, EWMA and instantaneous single-link throughput. Due to a different choice of , a more stable (left) or agile (right) reconfiguration is accomplished taking up two or three burst timeslots respectively.

an RSSI decrease by the same amount in Fig 6b does result in a more agile re-configuration of two timeslots taking up $60.28 \mathrm{~ms}$. After re-configuration, the EWMA filter is reset to the default value of $-67.5 \mathrm{dBm}$ in the middle of the hysteresis guard band. These results show that with the above switching mechanism, a re-configuration within two timeslots is possible, depending on the EWMA filter configuration and signal strength variation. In this way, our algorithm is useful in heterogeneous environments with diverse applications. It can cope with both long and short distance links, offers a solution to integrate applications with diverse requirements and provides robustness against channel gain variations since these variations have a direct impact on the RSSI metric. As such, a more suitable PHY will be selected depending on the current channel gain. Moreover, with a smart EWMA 


\begin{tabular}{lll}
\hline Hops & 50 kbps & 1 Mbps \\
\hline 1 hop & 3 & 10 \\
2 hops & 12 & 5 \\
3 hops & 1 & 0 \\
\hline
\end{tabular}

TABLE VIII: Number of hops to the coordinator with used PHY

configuration, it is achievable to favour more reliable PHYs to enhance robustness.

\section{E. Topology}

To assess the topology impact of adaptive multi-PHY $\mathrm{TSCH}$, our proof-of-concept implementation was established in an office environment spanning three floors of $805 \mathrm{~m}^{2}$ separated with reinforced concrete and making use of 33 Zolertia RE-motes on the imec Wireless Officelab, depicted in Fig77 To exclude RPL misbehaviour due to the use of multiple PHYs, all RPL traffic is sent using the default $50 \mathrm{kbps}$ PHY. After allowing the network to be initialized for 2 hours, from each node packets are being sent over the available unicast links to each parent to assess the quality of each link and determine if high speed $1 \mathrm{Mbps}$ would be possible. The resulting links between the nodes are shown in Fig7 7 , with links spanning over different floors indicated with an upwards or downwards arrow. As node 16 is a centrally positioned node on the 10th floor, it was chosen as the TSCH PAN coordinator and RPL root. Table VIII lists the number of hops that are needed to reach this coordinator. For each uplink path, the net PHY is also given, assuming $50 \mathrm{kbps}$ will be the bottleneck in a PHY-heterogeneous path. From these results it is clear that 15 out of 32 nodes are able to communicate with a max net throughput of $219.24 \mathrm{kbps}$, provided the burst link option is enabled while using the single-ACK timeslot structure. In contrast, these nodes would be limited to $31.32 \mathrm{kbps}$ in a pure $50 \mathrm{kbps}$ network. In addition, it is worth noting that many tree-based networks are limited in throughput since nodes closer to the gateway experience significantly higher congestion [15]. Since most high-throughput nodes are located close to the coordinator their increased throughput helps to reduce any buffer overflows or saturation. For communication between several floors, the more reliable $50 \mathrm{kbps}$ PHY is usually favoured to substantiate a smaller hop count to the coordinator, thereby reducing latency, and provide connectivity to hard to reach nodes due to its higher range. By using multiple PHYs in a TSCH network, we offer a solution to higher congestion in tree-based networks, provide an increased throughput towards the coordinator for almost half of the nodes in the network, and allow hard to reach nodes to connect to the network while keeping hop count (and latency) to a minimum. Moreover, our implementation is not bound to a single static network topology but can be used in heterogeneous areas by dynamically switching to the most suited PHY as seen in Fig 6 .

\section{FUTURE WORK}

Our research work and proof-of-concept implementation could be extended in several areas. First of all, the dynamic

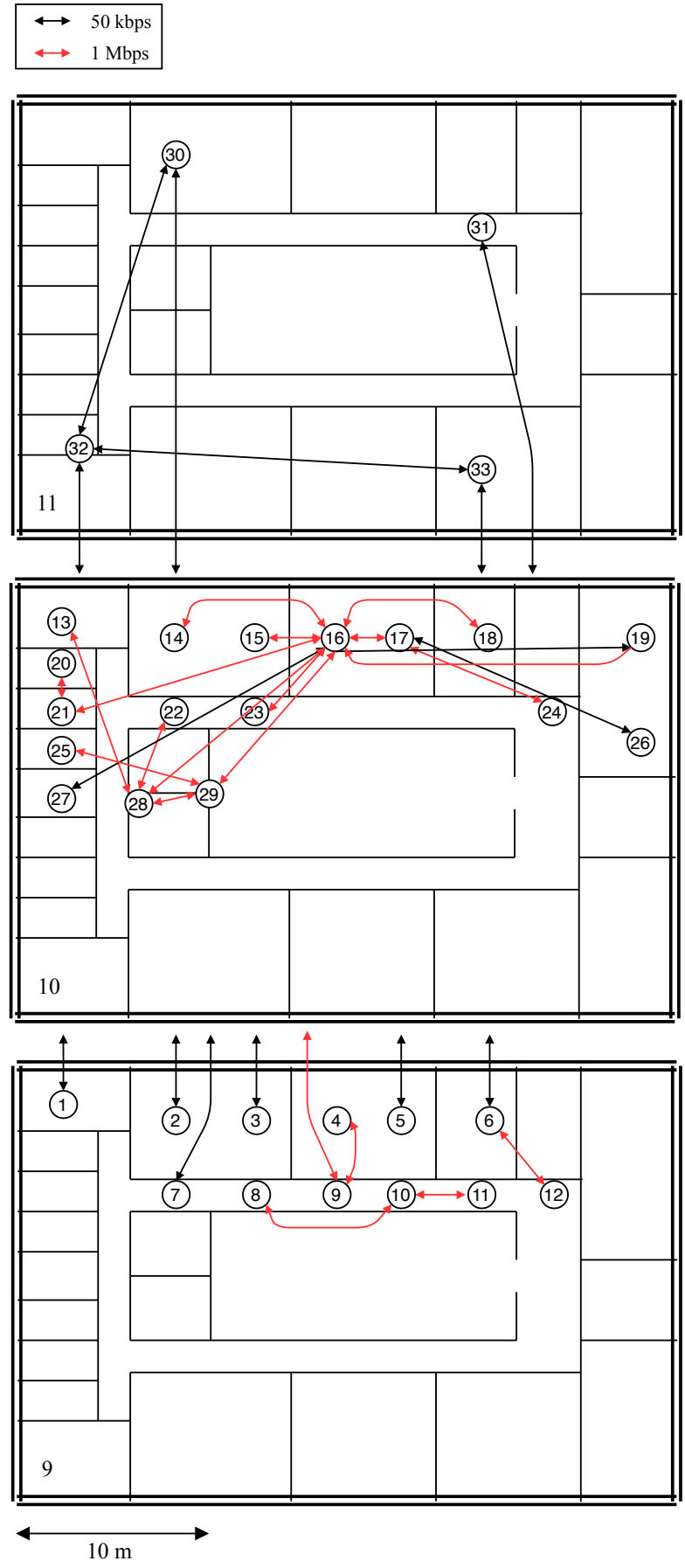

Fig. 7: Resulting network topology for 33 Zolertia RE-Motes across three floors. Node 16 at floor 10 is chosen to be the centrally positioned coordinator. Links spanning multiple floors are indicated with upwards or downwards arrows. 1 Mbps links are favoured withing the same floor, provided the distance is small, while $50 \mathrm{kbps}$ links are mostly used for inter-floor communication. As a result, a network can be formed over three floors with high throughput links if the link quality allows for it. 
link adaptation mechanism can be extended to allow more than two possible PHYs, as RSSI is only capable of distinguishing between a good and bad link and thus only two PHYs. As mentioned in Section IV, better metrics than RSSI can be evaluated and potentially even machine-learning techniques could be added. In this way, an optimal link adaptation metric can be chosen that maximizes end-to-end capacity. Secondly, as mentioned in [17], multi-PHY should be integrated in RPL by taking varying data rates and channel characteristics into account when calculating the Objective Function. Furthermore, the default Contiki-NG RPL protocol does not support mobility, and relies on custom modifications to support highly dynamic and/or mobile environments. The integration in RPL would allow RPL traffic not to be bound to a specific PHY and allow us to evaluate mobility in our final setup. With the above extensions, our research would be able to cover even more scenarios benefiting the adaptivity of multi-PHY TSCH networks.

\section{CONCLUSIONS}

In this paper we designed an adaptive sub-GHz multi-PHY TSCH MAC protocol, which can be used across existing schedulers assuming a fixed timeslot duration and is fully specification compliant. To optimally make use of the timeslot durations, two new multi-packet timeslot structures were defined using either multiple EACKs or a single EACK at the end of the timeslot. Both structures were evaluated theoretically in terms of throughput and energy consumption and we showed that using these structures has a positive impact on throughput and energy consumption. Furthermore, our results show that the throughput calculations can be experimentally verified with a $>99 \%$ accuracy. In case of limited queues, a choice can be made between both structures depending on the targeted scenario. Due to our flexible dynamic link adaptation mechanism, a different PHY can be allocated to each link depending on the current link quality. This mechanism can be used for two PHYs and the agility can be adjusted to fit certain proposed requirements. Finally, we used our proof-of-concept implementation in a real-world testbed with 33 Zolertia REMotes spanning three floors and covering $2550 \mathrm{~m}^{2}$. Our results show that by using adaptive multi-PHY TSCH a compact network can be established maximizing throughput depending on the environmental characteristics yet minimizing the number of hops by using both high throughput and reliable links. In addition, it provides a solution for higher congestion of nodes close to the root in tree-based networks.

\section{ACKNOWLEDGEMENTS}

This work was partially funded by the Flemish FWO SBO S004017N Intelligent DEnse And Longe range IoT networks (IDEAL-IoT) project. Part of this research was funded by the ICON project Internet of Shipping (IoS). IoS is realized in collaboration with imec, with project support from VLAIO. Project partners are imec, Exmar, Ovinto and Aloxy.

\section{REFERENCES}

[1] IEEE Standard for Telecommunications and Information Exchange Between Systems - LAN/MAN Specific Requirements - Part 15: Wireless Medium Access Control (MAC) and Physical Layer (PHY) Specifications for Low Rate Wireless Personal Area Networks (WPAN). IEEE Std 802.15.4-2003, pages 1-679, October 2003.

[2] IEEE Standard for Local and metropolitan area networks-Part 15.4: Low-Rate Wireless Personal Area Networks (LR-WPANs) Amendment 1: MAC sublayer. IEEE Std 802.15.4e-2012 (Amendment to IEEE Std 802.15.4-2011), pages 1-225, April 2012.

[3] IEEE Standard for Low-Rate Wireless Networks. IEEE Std 802.15.42015 (Revision of IEEE Std 802.15.4-2011), pages 1-709, April 2016.

[4] M. Y. Aalsalem, W. Z. Khan, W. Gharibi, M. K. Khan, and Q. Arshad Wireless Sensor Networks in oil and gas industry: Recent advances, taxonomy, requirements, and open challenges. Journal of Network and Computer Applications, 113:87-97, July 2018.

[5] Nouha Baccour, Anis Koubâa, Luca Mottola, Marco Antonio Zúñiga, Habib Youssef, Carlo Alberto Boano, and Mário Alves. Radio Link Quality Estimation in Wireless Sensor Networks: A Survey. ACM Transactions on Sensor Networks, 8(4), September 2012.

[6] F. Barać, M. Gidlund, and T. Zhang. Ubiquitous, Yet Deceptive: Hardware-Based Channel Metrics on Interfered WSN Links. IEEE Transactions on Vehicular Technology, 64(5):1766-1778, May 2015.

[7] M. Brachmann, S. Duquennoy, N. Tsiftes, and T. Voigt. IEEE 802.15.4 TSCH in Sub-GHz: Design Considerations and Multi-band Support. In IEEE Conference on Local Computer Networks (LCN), pages 42-50, October 2019.

[8] Glenn Daneels, Carmen Delgado, Steven Latré, and Jeroen Famaey. Towards Slot Bonding for Adaptive MCS in IEEE 802.15.4e TSCH Networks. In IEEE International Conference on Communications (ICC), pages 1-7, June 2020.

[9] Glenn Daneels, Esteban Municio, Bruno Van de Velde, Glenn Ergeerts, Maarten Weyn, Steven Latré, and Jeroen Famaey. Accurate Energy Consumption Modeling of IEEE 802.15.4e TSCH Using Dual-Band OpenMote Hardware. Sensors, 18(2):437, February 2018.

[10] D. Dujovne, T. Watteyne, X. Vilajosana, and P. Thubert. 6TiSCH: deterministic IP-enabled industrial internet (of things). IEEE Communications Magazine, 52(12):36-41, December 2014.

[11] Simon Duquennoy, Beshr Al Nahas, Olaf Landsiedel, and Thomas Watteyne. Orchestra: Robust Mesh Networks Through Autonomously Scheduled TSCH. In Proceedings of the ACM Conference on Embedded Networked Sensor Systems, page 337-350, November 2015.

[12] R.D. Gomes, P. Tuset-Peiró, and X. Vilajosana. Improving Link Reliability of IEEE 802.15.4g SUN Networks with Adaptive Modulation Diversity. Preprints, March 2020.

[13] T. Hamza and G. Kaddoum. Enhanced Minimal Scheduling Function for IEEE 802.15.4e TSCH Networks. In IEEE Wireless Communications and Networking Conference (WCNC), pages 1-6, April 2019.

[14] Bart Jooris, Jan Bauwens, Peter Ruckebusch, Peter De Valck, Christophe Van Praet, Ingrid Moerman, and Eli De Poorter. TAISC: A cross-platform MAC protocol compiler and execution engine. Computer Networks, 107:315-326, 2016.

[15] H. Kim, H. Kim, J. Paek, and S. Bahk. Load Balancing Under Heavy Traffic in RPL Routing Protocol for Low Power and Lossy Networks. IEEE Transactions on Mobile Computing, 16(4):964-979, April 2017.

[16] S. Kim, H. Kim, and C. Kim. ALICE: Autonomous Link-based Cell Scheduling for TSCH. In ACM/IEEE International Conference on Information Processing in Sensor Networks (IPSN), pages 121-132, April 2019.

[17] Jonathan Munoz, Xavier Vilajosana, and Tengfei Chang. Problem Statement for Generalizing 6TiSCH to Multiple PHYs. Internet Draft, Internet Engineering Task Force, July 2018. Work in Progress.

[18] M. Raza, N. Aslam, H. Le-Minh, S. Hussain, Y. Cao, and N. M. Khan. A Critical Analysis of Research Potential, Challenges, and Future Directives in Industrial Wireless Sensor Networks. IEEE Communications Surveys Tutorials, 20(1):39-95, March 2018.

[19] M. Saelens, J. Hoebeke, A. Shahid, and E. De Poorter. Impact of EU duty cycl and transmission power limitations for sub-GHz LPWAN SRDs: an overview and future challenges. EURASIP Journal on Wireless Communications and Networking, (1):219:1-219:32, September 2019.

[20] R. M. Sandoval, A. Garcia-Sanchez, and J. Garcia-Haro. Improving RSSI-Based Path-Loss Models Accuracy for Critical Infrastructures: A 
Smart Grid Substation Case-Study. IEEE Transactions on Industrial Informatics, 14(5):2230-2240, May 2018.

[21] E. Tanghe, W. Joseph, L. Martens, H. Capoen, K. Van Herwegen, and W. Vantomme. Large-scale fading in industrial environments at wireless communication frequencies. In 2007 IEEE Antennas and Propagation Society International Symposium, pages 3001-3004, June 2007.

[22] Texas Instruments. CC1200 Low-Power, High-Performance RF Transceiver, October 2014. SWRS123D, Rev. D.

[23] Pascal Thubert, Tim Winter, Anders Brandt, Jonathan Hui, Richard Kelsey, Phil Levis, Kristofer Pister, Rene Struik, Jp Vasseur, and Roger Alexander. RPL: IPv6 Routing Protocol for Low power and Lossy Networks. RFC 6550, Internet Engineering Task Force, May 2012.

[24] P. Tuset-Peiró, R.D. Gomes, P. Thubert, and X. Vilajosana. Evaluating IEEE 802.15.4g SUN for Dependable Low-Power Wireless Communications In Industrial Scenarios. Preprints, February 2020.

[25] M. Woehrle, M. Bor, and K. Langendoen. 868 MHz: A noiseless environment, but no free lunch for protocol design. In International Conference on Networked Sensing (INSS), pages 1-8, June 2012.

[26] Q. Zhang, Q. Zhang, W. Zhang, F. Shen, T. H. Loh, and F. Qin. Understanding the Temporal Fading in Wireless Industrial Networks: Measurements and Analyses. In International Conference on Wireless Communications and Signal Processing (WCSP), pages 1-6, October 2018. 